\title{
Captura por unidad de esfuerzo y estructura poblacional de la tortuga verde de Pacífico (Chelonia mydas) en el Golfo Dulce, Costa Rica
}

\author{
Didiher Chacón-Chaverri ${ }^{1}$, David A. Martínez-Cascante ${ }^{2}$, David Rojas ${ }^{1} \&$ Luis G. Fonseca $^{1}$ \\ 1. WIDECAST-Costa Rica, Tibás, San José, Costa Rica; dchacon@widecast.org, rojasmoralesd78@gmail.com, \\ luisfonsecalopez@gmail.com \\ 2. San Josecito de San Rafael, Heredia, Costa Rica; dawidh15@gmail.com
}

Recibido 11-VIII-2014. Corregido 20-XI-2014. Aceptado 22-XII-2014.

\begin{abstract}
Catch per unit effort and population structure of the Pacific green turtle (Chelonia mydas) in the Golfo Dulce, Costa Rica. The Golfo Dulce is one of the few tropical fjords in the world, and has recently been declared a Marine Area for Responsible Fisheries (AMPR), harboring mangrove ecosystems, coral reefs and seagrass beds, which are important feeding areas for green turtles (Chelonia mydas). In this study we estimate the catch per unit effort (CPUE) and population structure in a site in the western sector of the Golfo Dulce. Between August 2010 and March 2013, were captured a total of 253 green turtles (including 20 recaptures) using entanglement nets. The annual CPUE (CPUE, 1 unit: 100m of net for $7 \mathrm{~h}$ ) during the study ranged between 0.19 and 0.45 , decreasing gradually over the years, possibly prompted by mass mortality occurred in January 2013. We found that seasonality and temporality influence the CPUE according to the best-fit model. About $78.17 \%$ were considered adult females, with an average length of curved carapace (LCC) of $79.6 \pm 0.9 \mathrm{~cm}$. The female recaptured has been tagged previously in the Galapagos Archipelago. While none of the turtles tagged in the Golfo Dulce was reported nesting on any other beach. Our results suggest that the Golfo Dulce is an important area for green turtles, where individuals congregate for adults and subadults would feed on seagrasses and the fleshy parts of the mangroves. The area also represents a major challenge for international conservation, possibly because we captured adult females from nesting beaches of Panama and Colombia, which requires multilateral agreements that promote the recovery of the East Pacific green turtle. We suggest the continuation of this conservation project to use sea turtles as umbrella species to protect ecosystems in the Golfo Dulce. Rev. Biol. Trop. 63 (Suppl. 1): 363-373. Epub 2015 April 01.
\end{abstract}

Key words: Foraging grounds, entanglement nests, mangroves, sea grass.

La tortuga verde del Pacífico (Chelonia mydas) es una especie tropical, de hábitos costeros que anida principalmente en Michoacán (México) (Delgado-Trejo \& Alvarado-Díaz, 2012), en el Archipiélago de Galápagos (Ecuador) (Zárate \& Dutton, 2002) y en el Pacífico Norte de Costa Rica (Blanco et al., 2012; Fonseca et al., 2013.). Esta especie está catalogada como amenazada por la Unión Internacional para la Conservación (UICN) (UICN 2012) y se encuentra incluida en el Apéndice I de la Convención sobre el Comercio Internacional de Especies Amenazadas de Fauna y Flora Silvestres (CITES, 2012).
El conocimiento sobre las zonas de alimentación o congregación de la tortuga verde del Pacífico son desconocidos en Costa Rica. Normalmente las hembras llegan a poner sus huevos durante el periodo de anidación, y luego se desplazan a zonas de alimentación que pueden encontrarse a grandes distancias de su playa de anidación (Alvarado \& Figueroa, 1992). En el caso de la tortuga verde que anida en Costa Rica, se ha determinado que existen tortugas que se desplazan hasta el Golfo de Fonseca al norte de Nicaragua o al sur hasta el Estero de San Miguel, Panamá o permanecen de manera residente en el Pacífico Norte, frente 
al Golfo de Santa Elena, Guanacaste (Blanco et al., 2012).

La información derivada de transmisores satelitales muestra que las aguas de Costa Rica, son un corredor biológico importante para la tortuga verde del Pacífico durante su migración post-anidatoria (Blanco et al., 2012), ya que al menos una parte de la población del Archipiélago de Galápagos transitan por aguas costarricense para llegar hasta sitios de alimentación en Nicaragua y Panamá (Seminoff et al., 2008). Esto previamente había sido descrito con la recaptura de tres individuos de tortuga verde en el Pacífico Norte y Sur de Costa Rica, los cuales habían sido marcados en el Archipiélago de Galápagos (Green, 1984). Además, en aguas costarricenses se ha recapturado hembras marcadas en Michoacán, lo cual demuestra que en esta área se mezclan individuos de las dos poblaciones más importantes para esta especie (Alvarado \& Figueroa, 1992).

En el Pacífico Oriental, individuos juveniles y adultos principalmente se alimentan en áreas costeras, cercanas a estuarios, manglares, lagunas y pastos marinos (Bjorndal, 1997), como ha sido descrito para áreas de alimentación en Baja California y Bahía Magdalena (México) (López-Mendilaharsu, Gardner, Seminoff \& Riosmena-Rodríguez, 2005; Koch, Brooks \& Nichols, 2007) o en los alrededores de islas, tal como se ha documentado en el Parque Nacional Gorgona (Colombia) (Amorocho \& Reina, 2007). Su dieta consiste principalmente en algas rojas y verdes, sin embargo, también se alimentan de invertebrados y medusas (Seminoff, Resendiz, Nichols \& Chaloupka, 2003).

Para Costa Rica, la información histórica sobre sitios de anidación es pobre, únicamente existe información para Playa Naranjo, en donde se contabilizaron 80 hembras a inicios de los 70's (Cornelius, 1976), sin embargo, en los últimos años se han descubierto sitios importantes como Playa Nombre de Jesús, Playa Cabuyal, Playa Nancite e Isla San José, en donde la sumatoria anual del número de hembras supera los 500 individuos (Fonseca, Villachica, Matarrita \& Valverde, 2011; Blanco et al., 2012; Santidrián-Tomillo \& Hernández, 2012; Fonseca et., 2013), posicionando al Pacífico Norte de Costa Rica como el área de anidación más importante para esta especie en el istmo centroamericano.

No obstante, la información en Costa Rica sobre zonas de alimentación o rutas migratorias para la tortuga verde es limitada, máxime que los esfuerzos de investigación para esta especie se han enfocado en la protección de nidos y marcaje de hembras en las playas de anidación. Por tanto, este estudio tiene como objetivo principal la descripción de aspectos ecológicos de la tortuga verde en el sector oeste del Golfo Dulce, basados en la Captura por Unidad de Esfuerzo (CPUE) temporal y estacional, así como la descripción de la estructura de la población. Asimismo, se reporta una evaluación corporal de los individuos de acuerdo a lesiones externas y la presencia de fibropapilomas.

\section{MATERIALES Y MÉTODOS}

Área de estudio: El estudio se realizó en aguas costeras por encima de los $15 \mathrm{~m}$ de profundidad del sector oeste del Golfo Dulce, frente a Playa Blanca, la cual se ubica en el cantón de Osa de la Provincia de Puntarenas, Costa Rica $\left(8^{\circ} 38^{\prime} 51^{\prime \prime} \mathrm{N}-83^{\circ} 25^{\prime} 54^{\prime}\right.$ 'W) (Fig. 1). Este sector presenta una serie de desembocaduras de quebradas y riachuelos rodeados por manglares. En el sitio están presentes dos especies de pastos marinos: Halophyla sp. y Halodule sp.; así como tres especies de mangle: Rhizophora mangle, R. racemosa, Avicennia germinans.

El área registra una temperatura anual promedio de $26^{\circ} \mathrm{C}$, y un patrón de distribución de la precipitación bimodal, con un período de altas lluvias desde mayo hasta junio, y desde setiembre hasta noviembre, un período corto de bajas lluvias durante julio y agosto, y más pronunciado de diciembre a abril. El promedio de precipitación mensual desde el año 2000 ha oscilado entre los $3500 \mathrm{~mm}$ y $5500 \mathrm{~mm}$ (Instituto Meteorológico Nacional, Estación Meteorológica en Rincón de Osa). 


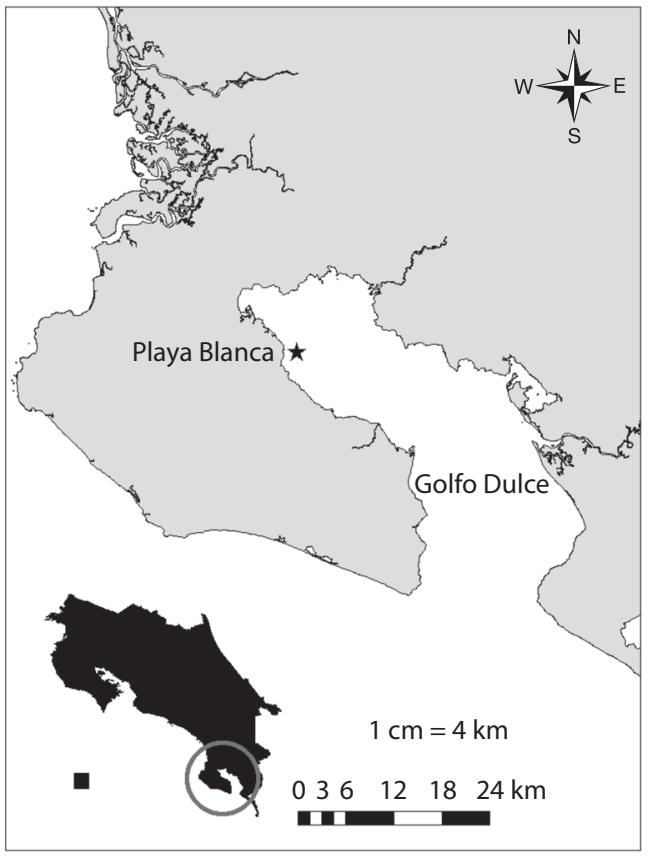

Fig. 1. Mapa de ubicación de Playa Blanca, Golfo Dulce, Costa Rica.

Fig. 1. Location map of Playa Blanca, Golfo Dulce, Costa Rica.

Desde el 2010, el Golfo Dulce es un sitio declarado por el Instituto Costarricense de Pesca y Acuacultura (INCOPESCA) como un Área Marina de Pesca Responsable (AMPR) y cuenta con una extensión de unos $750 \mathrm{~km}^{2}$, por lo que las actividades de pesca y extracción de recursos se encuentran reguladas por ley, bajo un modelo ambientalmente sostenible. Por tanto, se prohíbe la pesca de palangre, trasmallo y arrastre (Decreto Ejecutivo No 159, 17 de agosto de 2010).

Captura de las tortugas marinas y biometría del caparazón: Entre agosto de 2010 y marzo de 2013, se realizaron al menos 5 muestreos mensuales, en donde se capturaron tortugas marinas en el agua utilizando redes de enmalle $(100 \times 8 \mathrm{~m}$, luz de malla $=50 \mathrm{~cm})$ modificadas para capturar tortugas marinas y evitar la pesca incidental de otras especies. Las redes se colocaron únicamente durante el día, en periodos de $7 \mathrm{~h}$ (Seminoff et al., 2003). La distancia entre la red y la costa varió entre los 100 y $500 \mathrm{~m}$ de longitud, y entre los 3 y $10 \mathrm{~m}$ de profundidad, dependiendo de los movimientos de las mareas y la precipitación. Luego de capturar una tortuga, esta fue subida al bote y llevada a la orilla, en donde se marcó y midió. Posteriormente la tortuga se liberó en la zona donde fue capturada, dentro de un periodo no superior a los 30 minutos. Siempre se utilizó guantes de látex durante la manipulación de la tortuga, por previsiones de bioseguridad.

El largo curvo de caparazón (LCC) y el ancho curvo de caparazón (ACC) se midieron utilizando una cinta métrica flexible $( \pm 0.1 \mathrm{~cm})$, y cada medición se realizó por triplicado para verificar cada una de las medidas (Chacón, Sánchez, Calvo \& Ash, 2007). Después de realizar las mediciones, la tortuga se marcó externamente con marcas metálicas Inconel (Tipo 681, National Band \& Tag Company, Newport, $\mathrm{KY}$ ), específicamente sobre la segunda escama proximal de cada aleta delantera. Asimismo, desde enero de 2012 se colocó un PIT (Passive Integrated Transponder) en la aleta delantera derecha. Previo a la aplicación de las marcas metálicas, éstas fueron desinfectadas con Vanodine (Pfizer Inc.) para evitar infecciones en la piel de la tortuga e incrementar la coagulación de la sangre y la velocidad de cicatrización. En los casos en donde se observaron tortugas con cicatrices que hicieran suponer la pérdida de una marca metálica, se asumió que está tortuga era nueva, ya que pudo haber sido una hembra marcada en una playa anidación, por lo que no estaríamos duplicando el conteo.

Evaluación corporal: A cada tortuga capturada se le realizó una evaluación ocular para detectar lesiones, amputaciones, deformidades o la presencia de fibropapilomas. En los casos en donde se detectó alguna de estas características, está fue descrita en la hoja de campo. Por otra parte, se realizó una clasificación de cada tortuga de acuerdo al tamaño del LCC para determinar la estructura de la población. Se consideró a las tortugas que presentaban un $\mathrm{LCC} \geq 75 \mathrm{~cm}$ como adultos, mientras que los individuos que poseían una medición menor se 
clasificaron como juveniles, esto debido a que $75 \mathrm{~cm}$ de LCC es la menor medida reportada para una hembra anidando en Costa Rica (Fonseca et al., 2013).

CPUE y comparación temporal: La unidad de esfuerzo se definió como el despliegue de $100 \mathrm{~m}$ de la red de enmalle durante $7 \mathrm{~h}$, la CPUE se calculó dividiendo el número total de tortugas marinas capturadas en cada muestreo dividido por el número de unidades de esfuerzo.
CPUE esperada: Los datos de la CPUE se analizaron utilizando una distribución de Poisson inflada por ceros (PIC) (Wenger \& Freeman, 2008). Esta distribución incorpora un nuevo parámetro para separar la probabilidad de no haber observado individuos de la especie dado que se encontraban presentes (Ecuación 1), con la probabilidad de que no se encontrara ninguna tortuga en determinada unidad de muestreo.

$$
\operatorname{PIC}\left(y_{i} \mid \lambda_{i}, \psi_{i}\right)=\left\{\begin{array}{c}
y_{i}=0, \overbrace{\left(\psi_{i}-1\right)}^{\text {no presente }}+\overbrace{\psi_{i} \text { Pois }\left(0 \mid \lambda_{i} \cdot E\right)}^{\text {presente pero no observada }} \\
y_{i} \geq 1, \underbrace{\psi_{i} \operatorname{Pois}\left(y_{i} \mid \lambda_{i} \cdot E\right)}_{\text {presente }(y \text { individuos registrados })}
\end{array}\right.
$$

Donde y se refiere al conteo de individuos, $\lambda$ es la captura esperada por unidad de tiempo (individuos por hora), $\psi$ es la probabilidad de ocurrencia, $i$ se refiere a la $i$-ésima observación, y $E$ es el esfuerzo de muestreo. El valor del conteo esperado fue modelado con un predictor lineal (Ecuación 2), al igual que la probabilidad de ocurrencia (Ecuación 3). Las variables independientes consideradas fueron: el año desde el inicio del estudio, y la estación del año.

$$
\begin{gathered}
\lambda_{i}=\sum_{j=1}^{k} \beta_{j i} \cdot x_{j i} \\
\psi_{i}=\sum_{j=1}^{k^{\prime}} \beta_{j i}^{\prime} \cdot x_{j i}^{\prime}
\end{gathered}
$$

Donde $k$ es el número de parámetros en el predictor lineal de $\lambda, \mathrm{y} k$ ' es el número de parámetros en el predictor lineal de $\psi ; \beta$ son los parámetros del predictor lineal para el conteo esperado, y $\beta$ ' para la probabilidad de ocurrencia, y $x$ corresponde a la variable independiente para el conteo esperado, y $x$ ' para la ocurrencia. Los parámetros fueron estimados mediante la aproximación Bayesiana utilizando Cadenas de Markov de Monte Carlo (Monte Carlo Markov Chains, MCMC) (Gelman et al., 2004). Se definieron distribuciones previas no informativas, para lo cual se escogió la distribución Normal, con media igual a 0 , y varianza igual a $1 \times 10^{6}$.

La longitud de la MCMC, el periodo de quema y el adelgazamiento fueron obtenidos mediante el diagnóstico de Raftery-Lewis (Raftery \& Lewis, 1995). Se determinó que la cadena convergió a una distribución estacionaria si $\bar{R} \leq 1.1$. Para la comparación de modelos, se utilizó el criterio de pérdida predictiva, el cual se basa en la predicción y es independiente del número de parámetros incluidos en el modelo (Clark, 2007). La pérdida predictiva combina el concepto de exactitud (Ecuación 4) y precisión (Ecuación 5) al combinarse mediante el valor $D_{m}=G_{m}+P_{m}$. Donde $m$ corresponde a un modelo bajo evaluación. 


$$
\begin{gathered}
G_{m}=\sum_{i=1}^{n}\left(E\left[y_{i} \mid \mathrm{y}\right]-y_{i}\right)^{2} \\
P_{m}=\sum_{i=1}^{n} \operatorname{var}\left[y_{i} \mid \mathrm{y}\right]
\end{gathered}
$$

Todos los análisis estadísticos fueron realizados con el programa $\mathrm{R}$ versión 2.13.1 ( $\mathrm{R}$ Development Core Team 2013).

\section{RESULTADOS}

CPUE y retención de marcas: Entre agosto de 2010 y marzo de 2013 se capturaron un total de 253 tortugas empleando una unidad de muestreo total de 1078 (100m de red por 7h) (Cuadro 1). El CPUE promedio ha disminuido progresivamente a través del tiempo, encontrándose diferencias significativas entre los años (Fig. 2). No se encontraron diferencias significativas en el CPUE promedio entre la época seca y la época lluviosa (Fig. 2). La tasa de recaptura fue alta en los años 2011 y 2013, en contraste con lo registrado en el 2010

\section{CUADRO 1}

Estadísticas de captura de la tortuga verde (Chelonia mydas) en el Golfo Dulce, Costa Rica; incluyendo el numero de tortugas capturadas, número de recapturas, esfuerzo de muestreo y el promedio de la CPUE con sus respectivos intervalos de confianza al $95 \%$

\section{TABLE 1}

Catch statistics of the green turtle (Chelonia mydas) in the Golfo Dulce, Costa Rica, including the number of turtles caught, number of recaptures, sampling effort and average CPUE with their respective confidence intervals at $95 \%$

\begin{tabular}{cccccc} 
Año & \multicolumn{2}{c}{ Número de tortugas } & Tasa de recaptura (\%) & Esfuerzo de muestreo total & CPUE promedio \pm IC 95 \% \\
& Captura & Recaptura & 4.94 & 189 & $0.45 \pm 0.16$ \\
2010 & 81 & 4 & 14.71 & 105 & $0.37 \pm 0.16$ \\
2011 & 34 & 5 & 5.77 & 581 & $0.19 \pm 0.04$ \\
2012 & 104 & 6 & 14.71 & 203 & $0.19 \pm 0.11$ \\
2013 & 34 & 5 & 8.08 & 1078 & $0.25 \pm 0.50$ \\
\hline
\end{tabular}
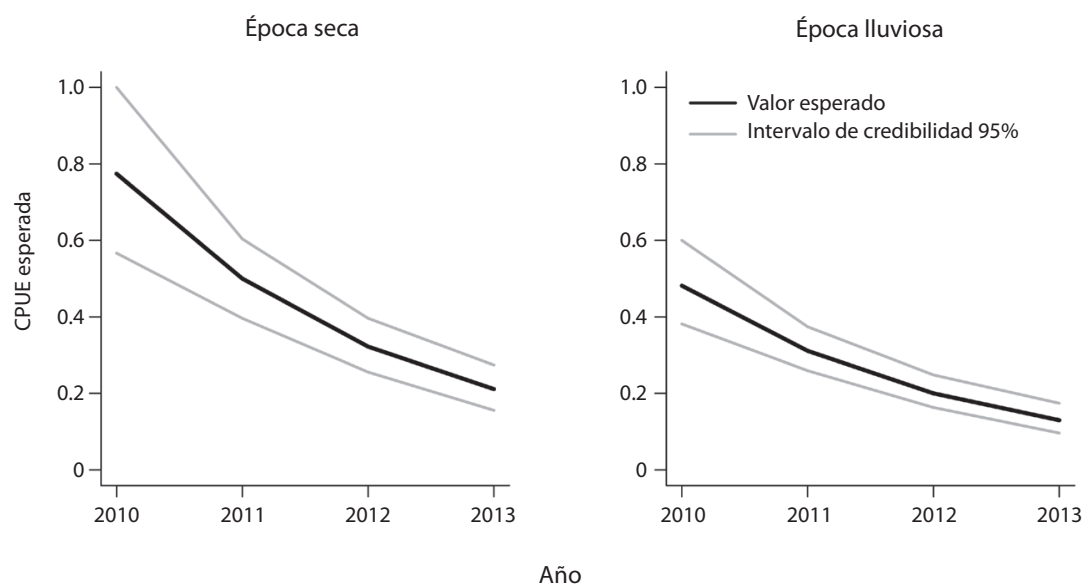

Fig. 2. CPUE esperada de tortuga verde (Chelonia mydas) basada en los parámetros del modelo de mejor ajuste. La temporada lluviosa en 2013 corresponde a una predicción del modelo.

Fig. 2. Expected CPUE green turtle (Chelonia mydas) from the parameters of the best fit model, including credible intervals at $95 \%$. The rainy season in 2013 corresponds to a model prediction. 
y 2012, en donde no superó el 6\%. El intervalo promedio de recaptura fue de 320.85 días (rango $=4-710, \mathrm{n}=20)$. Únicamente se recapturaron dos individuos en tres ocasiones, mientras que 18 tortugas se recapturaron una sola vez. De las 20 tortugas recapturadas, se observaron 12 que perdieron alguna de las marcas

\section{CUADRO 2}

Comparación de modelos basado en el criterio de pérdida predictiva para la CPUE esperada de tortuga verde (Chelonia mydas), en el Golfo Dulce, Costa Rica

\section{TABLE 2}

Model comparison based on the predictive loss criterion for expected CPUE green turtle (Chelonia mydas) in the Golfo Dulce, Costa Rica

\begin{tabular}{ccc} 
Modelo & Número de parámetros & $D_{m}$ \\
$\mathrm{p}(\mathrm{Y}+\mathrm{S}) \lambda(\mathrm{Y}+\mathrm{S})$ & 10 & 661.11 \\
$\mathrm{p}(\mathrm{S}) \lambda(\mathrm{Y}+\mathrm{S})$ & 5 & 672.09 \\
$\mathrm{p}(.) \lambda(\mathrm{Y}+\mathrm{S})$ & 4 & 672.29 \\
$\mathrm{p}(\mathrm{Y}) \lambda(\mathrm{Y}+\mathrm{S})$ & 7 & 678.30 \\
$\mathrm{p}(.) \lambda(\mathrm{Y})$ & 3 & 689.15 \\
$\mathrm{p}(\mathrm{S}) \lambda(\mathrm{Y})$ & 4 & 691.37 \\
$\mathrm{p}(\mathrm{Y}) \lambda(\mathrm{Y})$ & 6 & 700.45 \\
$\mathrm{p}(\mathrm{Y}) \lambda(\mathrm{S})$ & 6 & 750.22 \\
$\mathrm{p}(\mathrm{S}) \lambda(\mathrm{S})$ & 4 & 753.86 \\
$\mathrm{p}(.) \lambda()$. & 2 & 756.48 \\
$\mathrm{p}(\mathrm{Y}+\mathrm{S}) \lambda()$. & 8 & 756.60 \\
$\mathrm{p}(\mathrm{Y}) \lambda()$. & 5 & 761.68 \\
$\mathrm{p}(\mathrm{S}) \lambda()$. & 3 & 763.92 \\
\hline
\end{tabular}

Y: año; $\mathrm{S}$ : estación; p: probabilidad de ocurrencia; $\lambda$ : captura esperada por hora de esfuerzo. metálicas, lo que representa el $60 \%$ de los individuos recapturados. Siete correspondieron a la aleta derecha y cinco a la aleta izquierda.

CPUE esperada: Según el criterio de pérdida predictiva, el modelo con mejor ajuste indica que tanto la estación, como el año de estudio influyen sobre el conteo esperado y la probabilidad de ocurrencia (Cuadro 2). La estacionalidad y la tendencia temporal son importantes descriptores de la CPUE esperada, como indica su reiterada presencia dentro de los cuatro mejores modelos.

Estructura de la población: El LCC de las tortugas que se capturaron osciló entre 33.5 y $102.2 \mathrm{~cm}$ (LCC promedio $=79.6 \mathrm{~cm} \pm 0.9$ IC 95\%) (Cuadro 3). La distribución del tamaño de las tortugas verdes fue fuertemente sesgada hacia tortugas adultas, con $75.0-79.9 \mathrm{~cm}$ y 80.1 $84.9 \mathrm{~cm}$ como las clases dominantes (Fig. 3). El $69.09 \%$ de las tortugas consideradas como juveniles se ubicaron en la clase más cercana al tamaño mínimo de LCC de hembras adultas observadas anidando en Costa Rica. Un total de 55 tortugas fueron categorizadas como juveniles y 197 como adultos (Cuadro 2).

Evaluación corporal: Ninguna de las tortugas capturadas presentaron evidencia externa de fibropapilomas. Ninguna tortuga mostró traumas abiertos o lesiones en huesos, ojos y cloaca. Sin embargo, cinco individuos poseían alguna de sus aletas mutiladas con signos

CUADRO 3

Tortugas verdes (Chelonia mydas) capturadas en el Golfo Dulce, Costa Rica, entre agosto de 2010 y marzo de 2013, clasificados en individuos juveniles y adultos de acuerdo a la medida del LCC (cm)

TABLE 3

Green turtles (Chelonia mydas) captured in the Golfo Dulce, Costa Rica, between August 2010 and March 2013, categorized as juvenile and adult according to the LCC measurement (cm)

\begin{tabular}{ccccc} 
Año & \multicolumn{2}{c}{ Número de capturas } & \multicolumn{2}{c}{ Longitud $(\mathrm{cm})$} \\
& Juveniles & Adultos & LCC promedio \pm IC 95\% & Rango \\
2010 & 18 & 62 & $78.8 \pm 1.6$ & $53.5-91.8$ \\
2011 & 4 & 30 & $81.5 \pm 2.8$ & $53.0-95.5$ \\
2012 & 24 & 80 & $80.1 \pm 1.5$ & $47.6-102.2$ \\
2013 & 9 & 25 & $77.9 \pm 3.4$ & $33.5-91.5$ \\
Total & 55 & 197 & $79.6 \pm 0.9$ & $33.5-102.2$ \\
\hline
\end{tabular}




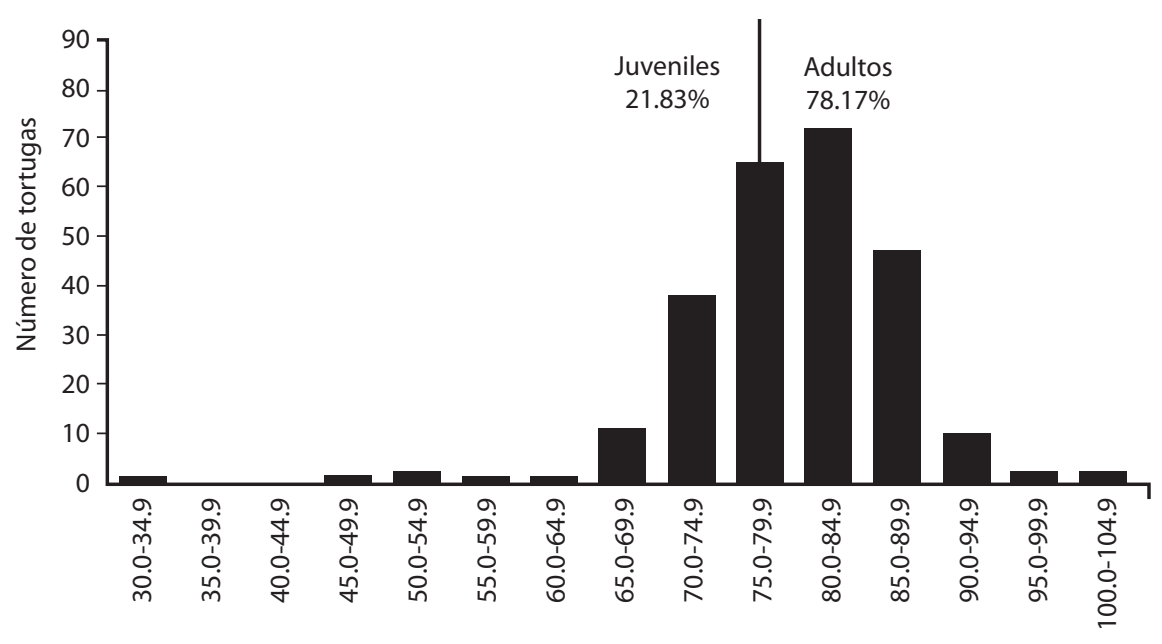

Largo curvo de caparazón $(\mathrm{cm})$

Fig. 3. Histograma del largo curvo de caparazón (LCC) de las tortugas verdes (Chelonia mydas) capturadas en el Golfo Dulce, Costa Rica entre agosto de 2010 y marzo de 2013. Solamente incluye las tortugas capturas en la primera ocasión, y se muestra la relación entre el porcentaje de juveniles y adultos capturados.

Fig. 3. Histogram of curved carapace length (CCL) of green turtles (Chelonia mydas) captured in the Golfo Dulce, Costa Rica between August 2010 and March 2013. Only includes turtle catches in the first time, and shows the relationship between the percentage of juveniles and adults captured.

evidentes de cicatrización, lo que representa el $1.98 \%$ de las tortugas capturadas. Ninguna tortuga capturada presentó lesiones luego de ser recapturada.

\section{Varamientos de tortugas y marcas metá-} licas recuperadas: Ninguna de las 253 tortugas marcadas en el Golfo Dulce fue reportada anidando o varadas en alguna playa de Costa Rica u otro país. En diciembre de 2010, se recapturó la tortuga con las marcas GAL3607-GAL3608, la cual había sido previamente marcada en la Quinta Playa, Archipiélago de Galápagos, el 16 de abril de 2010 (Parra, com. pers.). Posteriormente, esta misma tortuga se recapturó en marzo de 2011 y octubre de 2012. Entre el 20 y 31 de enero de 2013 se registró la muerte masiva de al menos 200 individuos de tortuga verde, los cuales fueron encontrados flotando en las aguas del interior del Golfo Dulce o en las orillas de las playas: Punta Banco, Zancudo y Pavones, todas al sur del cantón de Golfito. Ninguna de estas tortugas presentaba marcas metálicas en sus aletas delanteras (HerreraBejarano, 2013.

\section{DISCUSIÓN}

La CPUE en este estudio es considerablemente inferior al reportado en varios sitios del Pacífico de México como Punta Abreojos, Laguna San Ignacio, Laguna de Ojo de Liebre y Bahía Magdalena en donde la CPUE oscila entre 1 y 15 (Koch et al., 2007). Sin embargo, la CPUE es similar a lo observado en Baja California, México (rango=0.23-0.66) (Seminoff et al., 2003). Estas diferencias entre México y Costa Rica posiblemente se deban a que la costa Pacífica de México presenta al menos nueve playas de anidación que sobrepasan las 50 hembras temporada, lo que supera considerablemente lo que se contabiliza en Costa Rica, en donde como máximo se conocen cuatro sitios que alcancen este número de hembras (Isla San José, Coloradas, Cabuyal y Nombre de Jesús) (Blanco et al., 2012; Santidrián-Tomillo \& Hernández, 2012, Fonseca et al., 2013). Asimismo, en Playa Colola anidan más de 1000 tortugas al año (Delgado-Trejo \& Alvarado-Díaz, 2012), lo cual supera la sumatoria de todas las playas de anidación de Costa 
Rica, por lo que esperaríamos un mayor número de tortugas en las aguas mexicanas.

No obstante, llama fuertemente la atención que el CPUE en el Golfo Dulce ha mostrado una disminución progresiva a través de los años. A pesar del corto tiempo, lo anterior podría explicarse por cuatro razones: 1) las tortugas se han desplazado a otras áreas de alimentación (Bjorndal \& Bolten, 2010), 2) las tortugas están en los alrededores de la red de enmalle, pero casualmente no pasan en frente de la red, 3) las tortugas han migrado hacia sus playas de anidación, y 4) se ha dado una reducción en el número de tortugas presentes en el área como consecuencia de la pesca incidental (Dapp, Arauz, Spotila \& O'Connor, 2013). La segunda y tercera razón pueden que tengan más peso, ya que durante los muestreos realizados entre julio de 2012 y marzo de 2013 se ha notado que al momento de lanzar las redes y durante su constante revisión, se observan gran cantidad de tortugas respirando o tomando el sol, lo cual nos sugiere que las tortugas están en el área, pero que por alguna razón desconocida no están siendo capturadas por la red. Sin embargo, no podemos pasar por alto que la mortalidad masiva de tortugas verdes (al menos 200 individuos) que se presentó en enero de 2013 en el Golfo Dulce pudo mermar considerablemente a la especie en esta área.

Esta mortalidad presumiblemente fue consecuencia de la pesca incidental por palangre, y el uso de artes de pesca inapropiadas por estos mismos. A pesar de que el Golfo Dulce esta declarado como un AMPR por las autoridades del Gobierno de Costa Rica, no existe un control adecuado sobre la flota pesquera, y se está dando el uso de carnada viva en los anzuelos, lo que propicia que las tortugas por curiosidad se acerquen a las líneas y se enreden. Además, los palangres no están utilizando banderas para separar las líneas de pesca, sino más bien galones plásticos, los cuales quedan flotando y las tortugas se enredan en los mismos, propiciando el ahogamiento o la estrangulación de alguna extremidad como fue observado durante la realización de este estudio. No obstante, este estudio es de corta duración y no permite identificar una tendencia clara de la situación, pero la medición continua de este parámetro a largo plazo nos permitirá tener una visión más clara sobre el estado de la tortuga verde en el Golfo Dulce.

Estacionalmente encontramos que la probabilidad de ocurrencia es mayor en época lluviosa con excepción del 2010. Esto es similar a lo documentado en Bahía Magdalena en donde la CPUE fue superior en un $60 \%$ entre el verano y el invierno (Koch et al., 2007). Obviamente, Costa Rica al estar geográficamente ubicado en una zona más tropical que México, no presenta fluctuaciones extremas en la temperatura del aire y el mar, lo que hace que las tortugas puedan realizar todas sus funciones vitales con normalidad durante todo el año. En cambio, en México las tortugas bajan considerablemente su metabolismo o se desplazan a áreas con condiciones más cálidas (Felger, Cliffton \& Regal, 1976; Koch et al., 2007). Sin embargo, en el Golfo Dulce la disponibilidad de alimento es alta durante todo el año. En este sitio se presentan importantes bosques de mangle, en los cuales las tortugas se alimentan de los tallos suaves de los árboles que no sobrepasan los $75 \mathrm{~cm}$ de alto (Fonseca, Rojas, Volkart, Chacón \& Chacón-Chaverri, 2014). Dada la importancia de este bosque para la tortuga verde, se ha iniciado desde enero de 2012 un programa de reforestación del manglar, propiciando la recuperación de áreas que han sido deforestadas por los seres humanos (Pizarro \& Ángulo, 1993). Asimismo, durante todo el año las tortugas disponen de áreas con dos especies de pastos marinos (Halophyla sp. y Halodule sp.) de los cuales alimentarse, como fue documentado durante la realización de este estudio (Chacón-Chaverri, com pers.).

Por otra parte, se ha observado una fluctuación en la tasa de recaptura de las tortugas a través de los años. Esto concuerda con los observado en Bahía Magdalena, en donde este valor osciló entre 9-32\% (Koch et al., 2007). En general, la tasa de recaptura en el Golfo Dulce no debería de ser alta, ya que la mayor parte de los individuos capturados corresponden a ejemplares con tallas de adultos, por lo 
que si fueran hembras luego del periodo de alimentación, iniciarían su migración a las playas de anidación o a los sitios de cópula, los cuales están fuera del golfo. Asimismo, los machos también se moverían del golfo buscando sitios en donde se maximice la presencia de hembras en periodo reproductivo para poder copularlas y preservar sus genes. Esto normalmente ocurre frente a las playas de anidación; los machos no requieren de una alta inversión de energía en desplazarse grandes distancias, ya que disponen de un número importante de hembras en un área relativamente pequeña (Jensen, AbreuGrobois, Frydenberg \& Loeschke, 2006).

No se puede pasar por alto el hecho de que un $60 \%$ de las tortugas recapturadas perdieron alguna de sus marcas metálicas; sin embargo, estos individuos conservaron al menos una de las marcas, por lo que existe seguridad de no haber contado individuos en dos ocasiones. Como una medida complementaria para evitar el doble conteo, desde inicios del 2012 se comenzaron a aplicar marcas internas en cada uno de los individuos, siendo este tipo de registro imposible de perder, a menos de que la aleta de la tortuga sea amputada. Hasta el momento, no se ha reportado ninguna tortuga marcada en el Golfo Dulce en alguna playa de anidación, ni tampoco han sido encontradas muertas en playas o capturadas incidentalmente; lo cual mantiene un vacío de información sobre el origen de las tortugas que se congregan a alimentarse en el sitio. Posiblemente el único indicio es la recaptura de una tortuga proveniente del Archipiélago de Galápagos, Ecuador; la cual permaneció por 800 días en los alrededores del Golfo Dulce, luego de su etapa reproductiva. Normalmente la tortuga verde en el Archipiélago de Galápagos presenta un periodo post-anidatorio que oscila entre dos y seis años (Zárate, Parra \& Carrión, 2007), por lo que posiblemente esta tortuga este anidando ahora en Galápagos. Otro elemento que no permite conocer sobre la proveniencia de la tortuga verde en Golfo Dulce, es la escasa información que existe sobre esta especie en las playas del Pacífico de Panamá, ya que podrían existir sitios importantes de anidación en donde estén llegando estas tortugas.
En cuanto a la estructura de la población, nuestros resultados demuestran que el Golfo Dulce provee un hábitat importante para tortugas verdes adultas $(\mathrm{LCC} \geq 75 \mathrm{~cm})$. Solamente seis de los individuos capturados presentaron un LCC inferior a los $65 \mathrm{~cm}$ (2.37\%), lo cual es contrario a lo observado en Bahía Magdalena, Baja California y el Parque Nacional Gorgona, en donde el $90 \%$ de las tortugas capturadas corresponden a individuos inmaduros o juveniles (Seminoff et al., 2003; Amorocho \& Reina, 2007; Koch et al., 2007). Esta diferencia en las tallas capturadas no puede ser atribuida a las dimensiones de la luz de malla, ya que las redes utilizadas en los estudios en México cuentan con la misma dimensión. Inclusive, si en este estudio hubiéramos tomado como talla mínima reproductiva de las hembras lo observado en el Archipiélago de Galápagos ( $\mathrm{LCC}=66.0 \mathrm{~cm}$ ) (Zárate et al., 2007), más del 97\% de los individuos capturados se catalogarían como adultos.

Implicaciones en la conservación: Este estudio muestra por primera vez la importancia del Golfo Dulce para la protección y conservación de la tortuga verde, lo cual es un vacio de información que permitirá generar mejores medidas de manejo dentro del AMPR, tal como la zonificación del espacio marino y la gestión con las comunidades. Los resultados también demuestran que las tortugas que utilizan este sitio pueden incluso provenir del Archipiélago de Galápagos, lo cual implica una planificación a nivel internacional para promover medidas de conservación más efectivas. Asimismo, para maximizar estas acciones regionales, es necesaria la realización de un análisis genético de las tortugas muestreadas en el Golfo Dulce, ya que a través del ADN mitocondrial se podrían establecer el origen $\mathrm{u}$ orígenes de las tortugas que utilizan esta área. Por otra parte, consideramos de gran importancia la aplicación de las leyes en materia pesquera dentro del golfo, ya que prácticas ilegales y no registradas están dando lugar a la pesca incidental de tortugas, mermando fuertemente a las diferentes especies. Este trabajo debe de ser llevado a cabo de manera integral entre el Ministerio de Ambiente y 
Energía (MINAE) y el INCOPESCA, basado en la información científico - técnica derivada de este estudio. Finalmente, creemos que la recuperación de los hábitats críticos para la tortuga verde, como son los pastos marinos y los manglares permitirá a un mediano plazo la recuperación de esta especie en el Pacífico Oriental Este.

\section{AGRADECIMIENTOS}

Agradecemos el apoyo financiero de la NOAA, JFF, PTES, NFWF, Eco Teach, NEAQ, NAIB y ISV. Asimismo, agradecemos a los cientos de voluntarios que participaron en este estudio, sin los cuales no hubiera sido posible la realización del mismo. Este estudio se realizó bajo los permisos de investigación AJDIP/127-2011, AJDIP/227-2011, AJDIP/250-2011 y AJDIP/481-2012 otorgados por la Secretaría Técnica del INCOPESCA. Se agradece el Proyecto Consolidación de Áreas Marinas Protegidas del Sistema Nacional de Áreas de Conservación (SINAC), Programa de Naciones Unidas para el Desarrollo (PNUD) y el Global Environment Facility (GEF) por su colaboración en la publicación.

\section{RESUMEN}

El Golfo Dulce es uno de los pocos fiordos tropicales del mundo, y recientemente ha sido declarado como un Área Marina de Pesca Responsable (AMPR), albergando ecosistemas de manglar, corales y pastos marinos que son áreas de alimentación importantes para la tortuga verde (Chelonia mydas). En este estudio estimamos la captura por unidad de esfuerzo (CPUE) y la estructura de la población, en un sitio del sector oeste del Golfo Dulce. Entre agosto de 2010 y marzo de 2013, se capturaron un total de 253 tortugas verdes (incluyendo 20 recapturas) utilizando redes de enmalle. La CPUE anual (CPUE, 1 unidad: 100m de red por $7 \mathrm{~h}$ ) durante el estudio osciló entre 0.19 y 0.45 , reduciéndose progresivamente a través de los años, posiblemente propiciado por una mortalidad masiva ocurrida en enero de 2013. Se encontró que la estacionalidad y la temporalidad influyen en la CPUE de acuerdo al modelo de mejor ajuste. Cerca del $78.17 \%$ de las tortugas capturadas fueron consideradas como adultas, con un promedio de largo curvo de caparazón (LCC) de $79.6 \pm 0.9 \mathrm{~cm}$. Se recapturó una hembra que había sido previamente marcada en el Archipiélago de Galápagos. Mientras que ninguna de las tortugas marcadas en el Golfo Dulce fue reportada anidando en alguna playa. Nuestros resultados sugieren que el Golfo Dulce es un área importante para la tortuga verde, en donde se congregan individuos adultos o subadultos para alimentase de los pastos marinos y las partes carnosas de los mangles. Asimismo, el área representa un gran reto para la conservación internacional, ya que posiblemente las hembras adultas que capturamos provienen de playas de anidación de Panamá y Colombia, lo que requiere de acuerdos multilaterales que propicien la recuperación de la tortuga verde del Pacífico Este. Sugerimos la continuación de este proyecto de conservación para utilizar a las tortugas marinas como especie sombrilla para la protección de los ecosistemas del Golfo Dulce.

Palabras clave: Áreas de alimentación, redes de enmalle, manglares, pastos marinos.

\section{REFERENCIAS}

Alvarado, J., \& Figueroa, A. (1992). Recapturas PostAnidatorias de Hembras de Tortuga Marina Negra (Chelonia agassizii) Marcadas en Michoacán, México. Biotropica, 24, 560-566.

Amorocho, D. F., \& Reina, R. D. (2007). Feeding ecology of the East Pacific green sea turtle Chelonia mydas agassizii at Gorgona National Park, Colombia. Endangered Species Research, 3, 43-51.

Bjorndal, K. A. (1997). Foraging ecology and nutrition of sea turtles. Pp. 199-231. In P. L. Lutz \& J. L. Musick (Eds.), The biology of sea turtles (pp. 199-231). Boca Raton, USA.: CRC Press.

Bjorndal, K. A., \& Bolten A. B. (2010). Hawksbill sea turtles in seagrass pastures: success in a peripheral. Marine Biology, 157, 135-145.

Blanco, G. S., Morreale S. J., Bailey, H., Seminoff, J. A., Paladino, F. V., \& Spotila, J. R. (2012). Postnesting movements and feeding grounds of a resident East Pacific green turtle Chelonia mydas population from Costa Rica. Endangered Species Research, 18, 233-245.

Chacón, D., Sánchez, J., Calvo, J. J., \& Ash, J. (2007). Manual para el manejo y la conservación de las tortugas marinas en Costa Rica; con énfasis en la operación de proyectos en playa y viveros. San José, Costa Rica: Sistema Nacional de Áreas de Conservación (SINAC), Ministerio de Ambiente y Energía (MINAE).

CITES. (2012). Convención sobre el Comercio Internacional de Especies Amenazadas de Fauna y Flora Silvestres. Apéndice I, II y III. Ginebra, Suiza. Disponible en http://www.cites.org

Clark, J. S. (2007). Models for ecological data: an introduction. New Jersey, USA: Princeton University Press. 
Cornelius, S. E. (1976). Marine Turtle Nesting Activity at Playa Naranjo, Costa Rica. Brenesia, 8, 1-25.

Dapp, D., Arauz, R., Spotila, J. R., \& O’Connor, M. P. (2013). Impact of Costa Rican longline fishery on its bycatch of sharks, stingrays, bony fish and olive ridley turtles (Lepidochelys olivacea). Journal of Experimental Marine Biology and Ecology, 448, 228-239.

Delgado-Trejo, C., \& Alvarado-Díaz, J. (2012). Current Conservation Status of the Black Sea Turtle in Michoacán, México. In J. A. Seminoff \& B. P. Wallace (Eds.), Sea Turtles of the Eastern Pacific: Advances in Research and Conservation (pp. 236-278). Tucson: The University of Arizona Press.

Felger, R. S., Cliffton, K., \& Regal, P. J. (1976). Winter dormancy in sea turtles: independent discovery and explotation in the Gulf of California, México by two local cultures. Science, 191, 283-285.

Fonseca, L. G., Villachica, W. N., Matarrita, R. E, \& Valverde, R. A. (2011). Reporte final de la anidación de tortuga verde (Chelonia mydas), Playa Nancite, Parque Nacional Santa Rosa, Costa Rica (Temporada 2010-2011) (Informe Técnico). Guanacaste, Costa Rica.

Fonseca, L. G., Quirós, W., Villachica, W. N., Mora, J., Heidemeyer, M., \& Valverde, R. A. (2013). Anidación de tortuga verde (Chelonia mydas) del Pacifico, en la Isla San José, Área de Conservación Guanacaste, Costa Rica (Temporada 2012-2013) (Informe Técnico). Costa Rica: Institute for Tropical Studies Field Studies.

Fonseca, L. G., Rojas, D., Volkart, C., Chacón, D., \& Chacón-Chaverri, D. (2014). Reporte Final del proyecto de reforestación de manglares en Playa Blanca, Costa Rica (Diciembre 2012-Febrero 2014) (Informe Técnico). Costa Rica: WIDECAST.

Gelman, A., Carlin, J. B., Stern, H. S., Rubin, D. B., Chatfield, C., Tanner, M., \& Zidek, S. (2004). Bayesian data analysis. Florida, USA.: Chapman \& Hall/CRC.

Green, D. (1984). Long-Distance Movements of Galapagos Green Turtles. Journal of Herpetology, 18, 121-130.

Herrera-Bejarano, D. (2013). Necropsias y estudios postmorten realizados en 3 tortugas marinas (Informe Técnico). Costa Rica: WIDECAST.

Jensen, M. P., Abreu-Grobois, F. A., Frydenberg, F., \& Loeschke, V. (2006). Microsatellites provide insight into contrasting mating patterns in arribada vs. nonarribada olive ridley sea turtle rookeries. Molecular Ecology, 15, 2567-2575.

Koch, V., Brooks, L. B., \& Nichols, W. J. (2007). Population ecology of the green/black turtle (Chelonia mydas) in Bahía Magdalena, Mexico. Marine Biology, 153, 35-46.

López-Mendilaharsu, M., Gardner, S. C., Seminoff, J. A., \& Riosmena-Rodríguez, R. (2005). Identifying critical foraging habitats of the green turtle (Chelonia mydas) along the Pacific coast of the Baja California peninsula, Mexico. Aquatic Conservation: Marine and Freshwater Ecosystem, 15, 259-269.

Pizarro, F., \& Angulo, H. (1993). Diagnóstico de los manglares de la costa Pacífica de Costa Rica: Informe para la Comisión Nacional de Manglares. Costa Rica: UICN.

R Development Core Team. (2013). R: A language and environment for statistical computing. R Foundation for Statistical Computing. Vienna, Austria. ISBN 3-900051-07-0.

Raftery, A. E., \& Lewis, S. M. (1995). The number of iterations, convergence diagnostics and generic Metropolis algorithms. In W. R. Gilks, D. J. Spiegelhalter \& S. Richardson (Eds.), Practical Markov Chain Monte Carlo (pp. 1-15). London: Chapman \& Hall.

Santidrián-Tomillo, P., \& Hernández, R. (2012). Análisis de la Anidación de Tortugas Marinas en Playa Cabuyal y Golfo de Papagayo Sur (Informe Técnico). Guanacaste, Costa Rica.

Seminoff, J. A., Jones, T. T., Resendiz, A., Nichols, W. J., \& Chaloupka, M. (2003). Monitoring green turtles (Chelonia mydas) at a coastal foraging area in Baja California, Mexico: multiple indices describe population status. Journal of the Marine Biological Association of the United Kingdom, 83, 1355-1362.

Seminoff, J. A., Zárate, P., Coyne, M., Foley, D. G., Parker, D., Lyon B. N., \& Dutton, P. H. (2008). Post-nesting migrations of Galápagos green turtles Chelonia mydas in relation to oceanographic conditions: integrating satellite telemetry with remotely sensed ocean data. Endangered Species Research, 4, 57-72.

Unión Internacional para la Conservación de la Naturaleza (UICN). (2012). IUCN Red List of Threatened Species. IUCN Global Species Programme Red List Unit, Cambridge, UK. Retrived from http://www. iucnredlist.org/

Wenger, S. J., \& Freeman, M. C. (2008). Estimating species occurrence, abundance, and detection probability using zero-inflated distributions. Ecology, 89, 2953-29-59.

Zárate, P., \& Dutton, P. (2002). Tortuga verde. In E. Danulat \& G. J. Edgar (Eds.), Reserva Marina de Galápagos. Línea base de la biodiversidad (pp. 305-323). Parque Nacional Galápagos, Santa Cruz, Ecuador: Fundación Charles Darwin.

Zárate, P., Fernie, A., \& Dutton, P. (2003). First results of the East Pacific green turtle, Chelonia mydas, nesting population assessment in the Galapagos Islands. In J. A. Seminoff (Ed.), Proceedings of the TwentySecond Annual Symposium on Sea Turtle Biology and Conservation (pp. 70-73). USA: NOAA Technical Memorandum NMFS-SEFSC-503.

Zárate, P., Parra, M., \& Carrión, J. (2007). Informe Final Proyecto Anidación de la Tortuga Verde Chelonia mydas, durante la Temporada de Anidación 20062007. Parque Nacional Galápagos, Santa Cruz, Ecuador: Fundación Charles Darwin. 
